20

\title{
Коррекция флуоресцентных изображений биотканей на эффекты поглощения и рассеяния в них света
}

\author{
(C) С.А. Лысенко \\ Институт природопользования НАН Беларуси, \\ 220114 Минск, Беларусь \\ e-mail: lysenkorfe@gmail.com
}

Поступила в редакцию 06.02.2018 г.

В окончательной редакции 19.03.2018 г.

\begin{abstract}
Предложена методика коррекции влияния процессов ослабления света в биологических тканях на их флуоресцентные изображения. Передаточная функция, учитывающая потери в среде излучения на длинах волн возбуждения и испускания флуоресценции, рассчитывается в аналитическом виде в зависимости от транспортного показателя рассеяния и гемоглобинового индекса ткани. Последний определяется на основе цветного изображения ткани в отраженном видимом свете. Верификация разработанной методики проведена на основе компьютерного моделирования флуоресцентных изображений биоткани методом Монте-Карло.
\end{abstract}

DOI: $10.21883 / O S .2018 .07 .46276 .34-18$

\section{Введение}

Методы регистрации и анализа флуоресцентных изображений биотканей находят широкое применение в современной медицине для решения задач неинвазивной диагностики и фотодинамической терапии злокачественных опухолей, а также для визуализации их границ при оперативных вмешательствах [1-3]. Многие биологические молекулы, присутствующие в тканях человека (триптофан, флавины, никотинамид, коллаген, эластин, порфирины и др.), обладают способностью к флуоресценции при их возбуждении светом ультрафиолетового и синего диапазонов спектра, причем интенсивность и спектральный состав флуоресценции существенно зависят от состояния ткани $[4,5]$, что лежит в основе методов автофлуоресцентной диагностики. Для усиления контраста между опухолью и здоровой тканью пациенту вводится фотосенсибилизатор, способный избирательно накапливаться в злокачественных клетках и обеспечивать их яркую флуоресценцию, что составляет основу фотодинамической диагностики рака $[2,3]$.

Для эффективного детектирования опухолей внутриполостных органов разрабатывается эндоскопическая аппаратура с возможностью цифровой фото- и видеосъемки в режиме флуоресценции. Коммерчески доступные эндоскопические системы производителей KARL STORZ (Германия), Olympus (Япония) и др., адаптированные для работы с конкретным флуоресцентным красителем (индоцианин зеленый, протопорфирин IX и др.), позволяют наблюдать сенсибилизированную флуоресценцию обследуемой ткани в одном (достаточно широком) спектральном канале блока детектирования изображения на основе цветной или монохромной ПЗСкамеры [6]. Разрабатываются лабораторные макеты аппаратуры для мультиспектральной флуоресцентной съемки во время хирургических операций по удалению злокачественных опухолей с возможностью разделения вкладов эндогенных и экзогенных молекул-флуорофоров в регистрируемые изображения [7,8].

В то же время, несмотря на достаточно высокий уровень техники для флуоресцентной визуализации биотканей, имеется ряд нерешенных теоретических проблем, затрудняющих стандартизацию методов флуоресцентной диагностики и приводящих к недостаточно высокой чувствительности и специфичности выявления злокачественных опухолей. Одной из основных проблем является влияние поглощающих и рассеивающих свойств осматриваемой ткани на интенсивность и спектр ее флуоресценции. Как возбуждающее, так и флуоресцентное излучение поглощается хромофорами ткани и рассеивается на ее оптических неоднородностях. В результате этих процессов коэффициент связи между регистрируемым сигналом флуоресценции и концентрацией флуорофора в ткани зависит не только от характеристик измерительной аппаратуры, исключаемых путем калибровки, но и от параметров среды распространения излучения.

Существующие методы коррекции флуоресцентных изображений биотканей на эффекты поглощения и рассеяния в них света делятся на эмпирические и теоретические. Эмпирические методы основаны на вспомогательных измерениях сигналов отражения или автофлуоресценции исследуемой ткани и формировании из них корректирующих коэффициентов, зависящих от оптических параметров ткани схожим образом с сигналом ее сенсибилизированной флуоресценции [7-12]. Простейший метод коррекции флуоресцентного изображения ткани включает его нормировку на изображение в отраженном свете из спектральной области возбуждения флуоресценции $[9,10]$, что позволяет снизить разброс сигналов флуоресценции, вызванный поглощением тканью возбуждающего излучения. Для коррекции эффекта собственной флуоресценции ткани в спектральной области детектирования сигнала флуоресценции сенси- 
билизатора в $[11,12]$ предложено вышеотмеченное отношение сигналов сенсибилизированной флуоресценции и отражения нормировать на аналогичное отношение для автофлуоресценции, возбуждаемой вне зоны поглощения сенсибилизатора. Оба метода не компенсируют ослабление сигнала флуоресценции на длине волны еe испускания. Эмпирический учет таких искажений предполагает дополнительное освещение и регистрацию изображения биологического объекта в спектральной области его флуоресценции $[7,8]$, что ввиду различных динамических диапазонов сигналов отражения и флуоресценции существенно усложняет конструкцию диагностического устройства (необходимо разводить потоки отраженного и флуоресцентного излучения на камеры с различной чувствительностью).

Теоретические методы коррекции влияния индивидуальных оптических свойств биологических объектов на их флуоресцентные изображения предполагают использование моделей переноса излучения, в рамках которых рассчитываются передаточные функции среды на длинах волн возбуждения и испускания в ней флуоресценции. Наиболее надежные модели переноса излучения в оптически неоднородных средах построены на имитационном моделировании транспорта фотонов (метод Монте-Карло) [4,13]. Их главный недостаток необходимость разыгрывания большого количества случайных траекторий фотонов для получения статистически достоверных результатов. При коррекции флуоресцентных изображений, регистрируемых с высокой частотой кадров (например, при эндоскопии), оперативность расчетов передаточной функции среды имеет принципиальное значение. В связи с этим на практике гораздо большей популярностью пользуются оптические модели биологических объектов, построенные на аналитических решениях уравнения переноса излучения для различных асимптотических световых режимов внутри среды [14-18]. Для расширения области применения таких решений входящие в них величины часто параметризируются на основе более строгих численных решений $[19,20]$.

Отдельную проблему в теоретических методах коррекции флуоресцентных изображений представляет определение оптических параметров биологического объекта (показателей поглощения и рассеяния) в спектральных областях возбуждения и испускания им флуоресценции. Эту задачу можно решить, только располагая дополнительными средствами исследования объекта, например, на основе его многоспектральной съемки или структурированного освещения с изменяющейся пространственной частотой [21-25]. Реализовать такие измерения в условиях медицинского обследования внутриполостных органов и обеспечить при этом приемлемое качество флуоресцентных изображений в непрерывном видеорежиме непросто. В связи с этим актуален поиск новых простых и оперативных методов коррекции флуоресцентных изображений биотканей, которые могли бы быть реализованы на базе современной эндоскопической техники.

Известно, что основным поглощающим пигментом всех биотканей, содержащих сеть кровеносных сосудов, является гемоглобин, способный находиться в окисленной $(\mathrm{HHb})$ и в восстановленной $\left(\mathrm{HbO}_{2}\right)$ формах. Он оказывает значимое влияние на световой режим в среде практически для всех длин волн излучения (от ультрафиолетового до инфракрасного диапазонов включительно), поэтому коррекция его искажающего влияния на флуоресценцию биотканей представляет собой первоочередную задачу.

В работе [26] показана возможность использования трехцветных (RGB) изображений биотканей для определения их гемоглобинового индекса, под которым понимается отношение концентрации общего гемоглобина в ткани к ее транспортному показателю рассеяния на длине волны $632 \mathrm{~nm}$. Фактически этот индекс характеризует отношение поглощательной и рассеивающей способностей биоткани, а значит, и световой режим внутри среды. В настоящей работе предложена методика коррекции флуоресцентного изображения однородной биоткани на основе регрессионной связи корректирующей функции с гемоглобиновым индексом и транспортным показателем рассеяния ткани на длинах волн возбуждения $\lambda_{\mathrm{ex}}$ и испускания $\lambda_{\mathrm{em}}$ в ней флуоресценции. Параметры уравнения регрессии, зависящие от $\lambda_{\mathrm{ex}}$ и $\lambda_{\mathrm{em}}$, определены на основе численного моделирования переноса излучения в биоткани с учетом возможных вариаций ее спектрального показателя рассеяния, объемного содержания кровеносных сосудов и соотношения между содержанием окисленного и восстановленного гемоглобина в крови. Благодаря установленным зависимостям достигается возможность вычисления передаточной функции флуоресцирующего биообъекта в простом аналитическом виде (без необходимости решения уравнения переноса излучения) с использованием только лишь той информации, которая содержится в цветовой структуре изображения объекта. Эффективность предлагаемой методики оценивается на имитационных изображениях фантомов ткани с одинаковым содержанием флуорофора и с различным содержанием гемоглобина.

\section{Методика коррекции флуоресцентных изображений биотканей}

В коммерчески доступных эндоскопических системах для возбуждения сенсибилизированной флуоресценции обследуемой ткани используются ксеноновые или галогенные лампы, оснащенные узкополосным фильтром, характеристики которого оптимизированы для работы с конкретным сенсибилизатором [6]. Сигналы флуоресценции регистрируются в одном из цветовых каналов ПЗС-камеры, используемой для видеосъемки в отражен- 
ном белом свете, либо в дополнительном ИК канале высокочувствительной монохромной камеры.

В представлении биоткани моделью однородной полубесконечной среды поток флуоресцентного излучения, поступающий на фоточувствительные элементы ПЗС-матрицы, описывается следующим выражением $[4,5,16]$ :

$$
\begin{aligned}
F\left(\lambda_{\mathrm{ex}}, \lambda_{\mathrm{em}}\right)= & C_{f} \mathcal{E}_{f}\left(\lambda_{\mathrm{ex}}\right) Q_{f}\left(\lambda_{\mathrm{em}}\right) \\
& \times \int_{0}^{\infty} E\left(z, \lambda_{\mathrm{ex}}\right) T\left(z, \lambda_{\mathrm{em}}\right) d z,
\end{aligned}
$$

где $C_{f}-$ концентрация флуорофора в среде, $\varepsilon_{f}\left(\lambda_{\mathrm{ex}}\right)$ и $Q_{f}\left(\lambda_{\mathrm{em}}\right)$ - его показатель поглощения и квантовый выход флуоресценции, $\lambda_{\mathrm{ex}}$ и $\lambda_{\mathrm{em}}$ - длины волн, на которых флуорофор поглощает и испускает излучение, $E\left(z, \lambda_{\mathrm{ex}}\right)$ - поток возбуждающего излучения на глубине $z, T\left(z, \lambda_{\mathrm{em}}\right)$ - функция, характеризующая передачу мощности излучения от изотропного точечного источника на глубине $z$ к поверхности среды.

Интеграл в формуле (1) определяет передаточную функцию $D\left(\lambda_{\mathrm{ex}}, \lambda_{\mathrm{em}}\right)$, связывающую регистрируемый сигнал флуоресценции с концентрацией флурофора внутри среды. Для нахождения этой функции необходимо знать оптические параметры среды на длинах волн $\lambda_{\mathrm{ex}}$ и $\lambda_{\mathrm{em}}$ : показатель поглощения $\mu_{a}$, показатель рассеяния $\mu_{s}$ и средний косинус индикатрисы рассеяния $g$. Эти параметры, в свою очередь, зависят от структуры и компонентного состава биологической ткани, поэтому их оценки в принципе можно получать на основе спектроскопии диффузного отражения $[25,27,28]$. Однако при эндоскопическом исследовании тканей внутриполостных органов измерения их коэффициента диффузного отражения (с точностью до нормировочного множителя) возможны только в трех спектральных диапазонах, соответствующих синему, зеленому и красному приемным каналам ПЗС-матрицы. В этих условиях провести детальный анализ компонентного состава ткани не представляется возможным. Тем не менее, как следует из теории переноса излучения [29-31], коэффициент диффузного отражения среды с альбедо однократного рассеяния, близким к единице $\left(\mu_{s} \gg \mu_{a}\right)$, определяется преимущественно отношением ее показателя поглощения и транспортного показателя рассеяния $\mu_{s}^{\prime}=\mu_{s}(1-g)$. Для многих биотканей это условие в достаточной мере выполняется, особенно в спектральном окне их прозрачности (600-900 nm). Кроме того, учитывая, что основным поглощающим компонентом биоткани является гемоглобин, локализованный в кровеносных сосудах, отношение $\mu_{a} / \mu_{s}^{\prime}$ тесно коррелирует с введенным нами ранее гемоглобиновым индексом ткани, который можно оценить по цветовой структуре ее изображения [26].

Гемоглобиновый индекс (HbI) определяется как отношение $\left(f_{V} C_{\mathrm{Hb}}\right) / \mu_{s}^{\prime}(632 \mathrm{~nm})$, где $f_{V}-$ объемное содержание кровеносных сосудов в ткани, $C_{\mathrm{Hb}}=150 \mathrm{~g} / \mathrm{L}-$ концентрация общего ( $\mathrm{HHb}$ и $\left.\mathrm{HbO}_{2}\right)$ гемоглобина в крови. Карту пространственного распределения $\mathrm{HbI}$ в пределах поля зрения объектива камеры можно получить на основе анализа независимых отношений фотосигналов в ее цветовых каналах - $\rho_{1}$ (синий/зеленый) и $\rho_{2}$ (зеленый/красный). Такой анализ основан на использовании большого количества расчетных „обучающих“ данных, представленных в виде точек в трехмерной системе координат $\left(\rho_{1}, \rho_{2}, \mathrm{HbI}\right)$. Поверхность, проходящая через эти точки, аппроксимирована следующим уравнением [26]:

$$
\ln (\mathrm{HbI})=\sum_{k=0}^{3} \sum_{m=0}^{3} a_{k m}\left(\rho_{1}\right)^{k}\left(\rho_{2}\right)^{m},
$$

где $k+m \leq 3, a_{k m}$ - параметры аппроксимации, зависящие от спектральных характеристик источника непрерывного видимого света и средства приема изображения объекта. Массив „обучающих“ данных рассчитывается один раз для каждой съемочной системы на основе разработанной нами аналитической модели спектра диффузного отражения биоткани [32,33]. Погрешность таких расчетов не превышает 4\% в сравнении с методом Монте-Карло.

Рассмотрим связь HbI с передаточной функцией биоткани, которую определим следующим выражением:

$$
D\left(\lambda_{\mathrm{ex}}, \lambda_{\mathrm{em}}\right)=\int_{0}^{\infty} \frac{E\left(z, \lambda_{\mathrm{ex}}\right)}{E_{0}\left(\lambda_{\mathrm{ex}}\right)} T\left(z, \lambda_{\mathrm{em}}\right) d z,
$$

где $E_{0}\left(\lambda_{\text {ex }}\right)$ - освещенность ткани на длине волны возбуждения флуоресценции, определения других величин даны в пояснениях к формуле (1). Рассмотрение проведем в рамках ставшей уже стандартной модели однородной биоткани, многократно отработанной в экспериментах $[17,27,28,34]$. Модель описывает спектральные зависимости показателя поглощения $\mu_{a}$ и транспортного показателя рассеяния $\mu_{s}^{\prime}$ ткани следующими уравнениями:

$$
\begin{gathered}
\mu_{s}^{\prime}(\lambda)=\mu_{s}^{\prime}\left(\lambda_{0}\right)\left[\lambda_{0} / \lambda\right]^{v}, \\
\mu_{a}(\lambda)=\alpha_{V} f_{V} \mu_{a, b l}(\lambda)+\left(1-f_{V}\right) \mu_{a, t i s}, \\
\mu_{a, b l}(\lambda)=\frac{C_{\mathrm{Hb}}}{M_{\mathrm{Hb}}}\left[S \varepsilon_{\mathrm{HbO}_{2}}(\lambda)+(1-S) \varepsilon_{\mathrm{HHb}}(\lambda)\right], \\
\alpha_{V}=\left(1-\exp \left(-\mu_{a, b l} D_{V}\right)\right) /\left(\mu_{a, b l} D_{V}\right),
\end{gathered}
$$

где $\lambda_{0}=632 \mathrm{~nm}$ - условно выбранная длина волны, на которой задается опорное значение транспортного показателя рассеяния среды $\mu_{s}^{\prime}\left(\lambda_{0}\right) ; v-$ параметр степенной функции, аппроксимирующей зависимость $\mu_{s}$ от длины волны излучения; $f_{V}$ и $D_{V}-$ объемная концентрация и средний диаметр кровеносных сосудов, пронизывающих ткань; $\mu_{a, t i s}$ и $\mu_{a, b l}$ - показатели поглощения тканиосновы и крови; $\alpha_{V}-$ поправочный коэффициент к поглощательной способности среды, учитывающий эффект 
пространственной локализации поглотителей (крови в сосудах) и наличие свободных участков между поглощающими объемами [35]; $C_{\mathrm{Hb}}-$ концентрация гемоглобина в крови (полагается постоянной - $150 \mathrm{~g} / \mathrm{L}$ ); $M_{\mathrm{Hb}}=64500 \mathrm{~g} / \mathrm{mol}$ - молярная масса гемоглобина; $\varepsilon_{\mathrm{HbO}_{2}}$ и $\varepsilon_{\mathrm{HHb}}-$ молярные показатели поглощения окисленного и восстановленного гемоглобина, $S-$ степень насыщения гемоглобина кислородом.

Для расчета функции (3) будем использовать аналитический метод, предложенный в работе [25]. Метод удачно сочетает в себе ряд элементов теории переноса излучения: принцип взаимодействия элементарных слоев среды, соотношения инвариантности, асимптотические решения уравнения переноса излучения. Поле излучения в спектральном диапазоне возбуждения флуоресценции в среде описывается суперпозицией падающего направленного, падающего диффузного и отраженного диффузного потоков. Для описания светового поля в спектральном диапазоне флуоресцентного свечения среды используется двухпотоковое приближение. При этом в отличие от в двухпотоковой теории КубелкиМунка и четырехпотоковой модели переноса излучения для связи световых потоков на границах элементарных слоев среды не используются какие-либо эмпирические параметры, не связанные напрямую с фундаментальными характеристиками поглощения и рассеяния света в среде. Потоки флуоресценции внутри и на поверхности многослойной мутной среды вычисляются в матричном виде по заданным значениям показателя поглощения, показателя рассеяния, среднего косинуса индикатрисы рассеяния и толщины отдельных слоев среды.

Учитывая малый объем измерительной информации, реально доступной при исследованиях биотканей с помощью эндоскопических систем, расчеты передаточной функции $D\left(\lambda_{\mathrm{ex}}, \lambda_{\mathrm{em}}\right)$ выполнялись в рамках модели однородной полубесконечной среды с оптическими параметрами, задаваемыми формулами (4)-(7). Примеры таких расчетов приведены на рис. 1. Моделирование выполнено при трех значениях объемной концентрации сосудов в ткани: $f_{V}=0.01,0.02,0.05$. Для каждого значения $f_{V}$ рассмотрено 1000 случайных комбинаций других модельных параметров, равномерно распределенных в следующих диапазонах: $\mu_{s}^{\prime}\left(\lambda_{0}\right)=0.1-5.0 \mathrm{~mm}^{-1}$, $v=0-3, S=0.75-0.95$ [36-38]. Значения остальных параметров модели полагались фиксированными, поскольку учесть их влияние на световой режим в среде только лишь на основе анализа цветного изображения биоткани не представляется возможным [32,33]. К числу неизменяемых параметров относились диаметр сосудов $D_{V}=25 \mu \mathrm{m}$, показатель поглощения ткани-основы $\mu_{a, t i s}=0\left(\mu_{a, t i s} \ll \mu_{a, b l}\right)$, средний косинус индикатрисы рассеяния $g=0.8$ и показатель преломления ткани $n=1.38$

Результаты моделирования функции $D\left(\lambda_{\mathrm{ex}}, \lambda_{\mathrm{em}}\right)$, приведенные на рис. 1, дают представление о важности учета содержания гемоглобина в ткани при анализе ее флуоресцентного изображения. Видно, что вариации

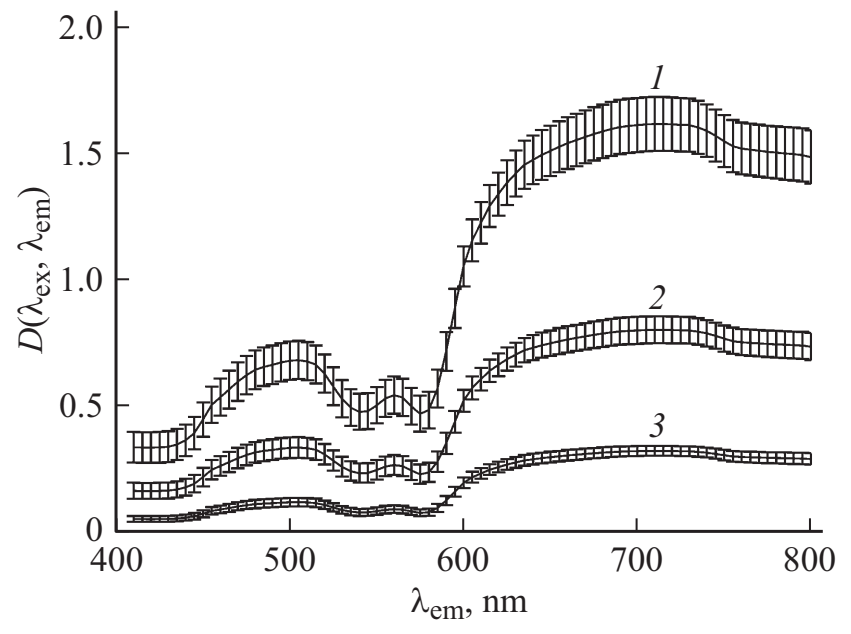

Рис. 1. Передаточная функция биологической ткани, рассчитанная на основе модели при $f_{V}=0.01(1), 0.02$ (2), 0.05 (3). Верхние и нижние границы точек на графиках соответствуют среднеквадратическому отклонению функции $D\left(\lambda_{\mathrm{ex}}, \lambda_{\mathrm{em}}\right)$ при вариациях модельных параметров $\mu_{s}^{\prime}\left(\lambda_{0}\right), v$ и $S$, ограниченных диапазоном значений коэффициента диффузного отражения среды $0.01-0.8$.

показателя рассеяния ткани проявляются в потоках выходящего из среды флуоресцентного излучения сравнительно слабо на фоне более мощного влияния на них гемоглобина. Учитывая, что сам показатель рассеяния для каждого типа биоткани изменяется в достаточно узких пределах [38], при коррекции флуоресцентных изображений исследуемой ткани в первом приближении можно использовать типичный для нее спектр $\mu_{s}^{\prime}(\lambda)$. Ниже будет показано, что влияние $\mu_{s}^{\prime}(\lambda)$ на результат коррекции регистрируемых сигналов флуоресценции при известном гемоглобиновом индексе биоткани сводится лишь к заданию их реперного уровня, что не сказывается на контрасте изображения и несущественно при анализе относительного распределения флуорофора в пределах осматриваемого участка ткани.

С целью достижения оперативности обработки изображений функцию $D\left(\lambda_{\mathrm{ex}}, \lambda_{\mathrm{em}}\right)$ полезно представить в простом аналитическом виде. Это можно сделать путем аппроксимации результатов описанного выше моделирования. Для устранения произвола в выборе случайных комбинаций модельных параметров и ограничения области вариаций моделируемых световых потоков на поверхности среды (при ее одной и той же освещенности) некоторыми разумными пределами необходимы дополнительные ограничивающие условия, учитывающие априорную информацию об исследуемом объекте. В наиболее мягкой форме эти условия могут быть сформулированы для коэффициента диффузного отражения биоткани $R$, значения которого редко выходят из диапазона 0.01-0.8. Комбинации модельных параметров, приводящие к значениям $R$ за пределами указанного диапазона, должны быть исключены из рассмотрения. 

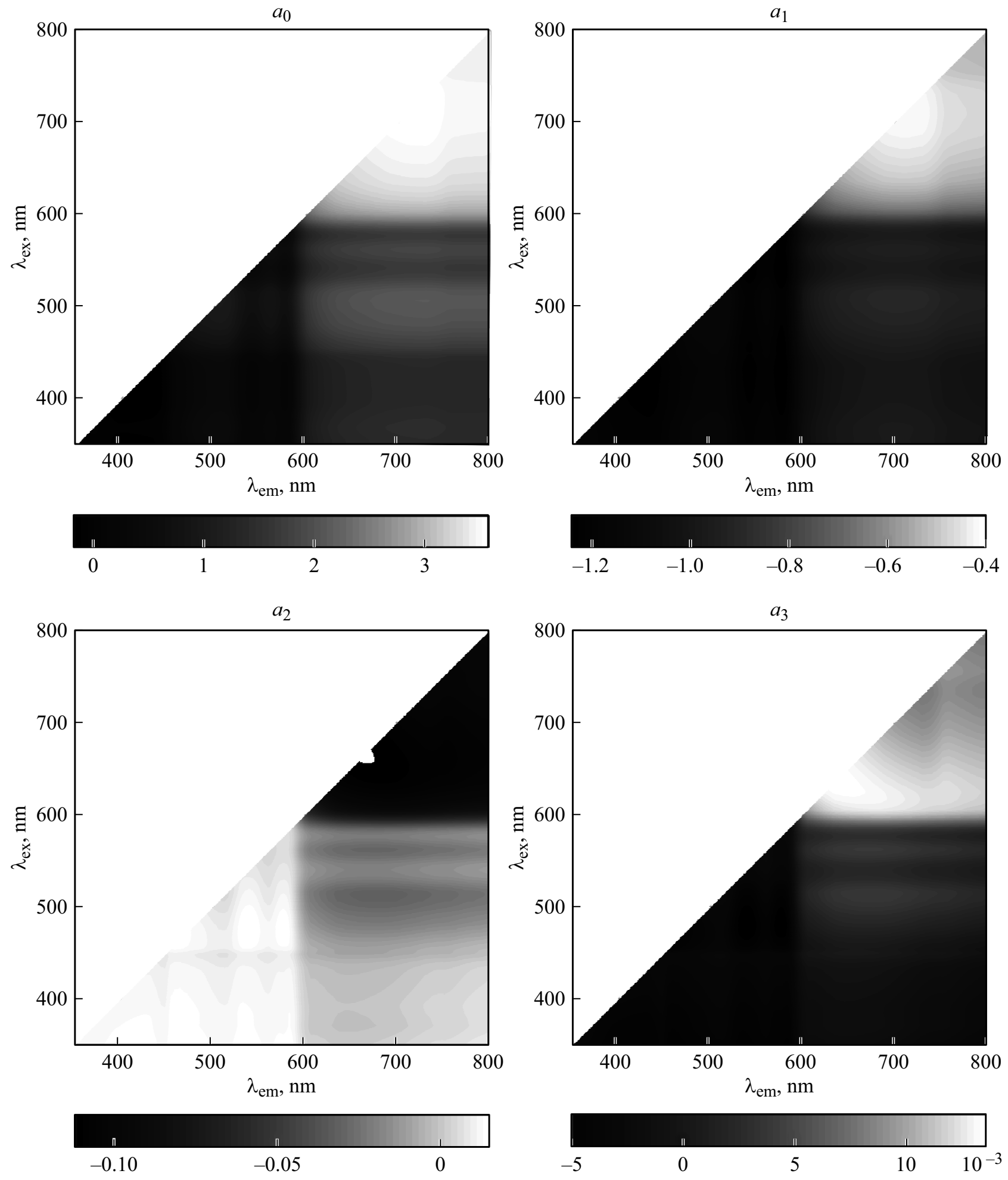

Рис. 2. Зависимости коэффициентов уравнения регрессии (8) от длин волн возбуждения и испускания флуоресценции.

Для контроля разыгрываемых комбинаций модельных параметров без существенных затрат на это машинного времени использовалась аналитическая модель зависимости $R\left(\mu_{a}, \mu_{s}^{\prime}, g, n\right)$, предложенная автором в работе [33].
Передаточная функция среды, имитирующей биоткань, рассчитана для $10^{4}$ случайно сгенерированных значений ее параметров: $f_{V}=0.002-0.2, \mu_{s}^{\prime}\left(\lambda_{0}\right)=$ $=0.1-5.0 \mathrm{~mm}^{-1}, v=0-3, S=0.75-0.95$. Разброс указанных параметров ограничен диапазоном вариаций ко- 
эффициента диффузного отражения среды $0.01-0.8$. Расчеты $D\left(\lambda_{\text {ex }}, \lambda_{\text {em }}\right)$ охватывают спектральные диапазоны $\lambda_{\mathrm{ex}}=350-800 \mathrm{~nm}$ и $\lambda_{\mathrm{em}}=\lambda_{\mathrm{ex}}-800 \mathrm{~nm} \mathrm{c} \mathrm{разрешением}$ в $1 \mathrm{~nm}$. Ансамбль данных, полученных для каждой пары $\lambda_{\mathrm{ex}}$ и $\lambda_{\mathrm{em}}$, аппроксимирован следующим уравнением:

$$
\begin{gathered}
\ln \left[D\left(\lambda_{\mathrm{ex}}, \lambda_{\mathrm{em}}\right)\right]=a_{0}\left(\lambda_{\mathrm{ex}}, \lambda_{\mathrm{em}}\right)+a_{1}\left(\lambda_{\mathrm{ex}}, \lambda_{\mathrm{em}}\right) \ln (\mathrm{HbI}) \\
+a_{2}\left(\lambda_{\mathrm{ex}}, \lambda_{\mathrm{em}}\right) \ln \left[\mu_{s}^{\prime}\left(\lambda_{\mathrm{ex}}\right)\right]+a_{3}\left(\lambda_{\mathrm{ex}}, \lambda_{\mathrm{em}}\right) \ln \left[\mu_{s}^{\prime}\left(\lambda_{\mathrm{em}}\right)\right]
\end{gathered}
$$

где $a_{0}, \ldots, a_{3}$ - спектрально зависящие коэффициенты, представленные в виде цветовых карт на рис. 2. При необходимости вычисления функции $D\left(\lambda_{\mathrm{ex}}, \lambda_{\mathrm{em}}\right)$ в конечных спектральных интервалах $\left[\lambda_{1}, \lambda_{2}\right]$, соответствующих приемным каналам съемочного устройства, можно воспользоваться известными квадратурными формулами для нахождения интегральной передаточной функции по ее монохроматическим значениям, либо исходить из формулы (8) со средневзвешенным значением $\lambda_{\mathrm{em}}$ :

$$
\bar{\lambda}_{\mathrm{em}}=\frac{\int_{\lambda_{1}}^{\lambda_{2}} \lambda Q_{f}(\lambda) \Phi(\lambda) d \lambda}{\int_{\lambda_{1}}^{\lambda_{2}} Q_{f}(\lambda) \Phi(\lambda) d \lambda},
$$

где $Q_{f}$ - квантовый выход флуорофора, $\Phi-$ спектральная чувствительность фотоприемного устройства.

Относительная среднеквадратическая погрешность аппроксимации функции $D\left(\lambda_{\mathrm{ex}}, \lambda_{\mathrm{em}}\right)$ уравнением (8) приведена на рис. 3. Поскольку формула (8) не учитывает газовый состав гемоглобина (ввиду ограниченных информационных возможностей рассматриваемых измерений), то наибольшие ее погрешности соответствуют длинам волн $\lambda_{\text {ex }}$ и $\lambda_{\text {em, }}$ при которых молярный показатель поглощения $\mathrm{HHb}$ значительно превосходит аналогичный показатель для $\mathrm{HbO}_{2}$. Наименьшие погрешности формула (8) дает при использовании в качестве $\lambda_{\mathrm{ex}}$ и $\lambda_{\mathrm{em}}$ точек пересечения спектров поглощения $\mathrm{HHb}$ и $\mathrm{HbO}_{2}$, для которых общий показатель поглощения крови не зависит от степени ее насыщения кислородом.

Таким образом, предлагаемая методика коррекции флуоресцентных изображений биотканей включает следующие этапы:

- регистрацию RGB-изображения ткани при ее освещении непрерывным источником видимого света;

- преобразование составляющих цветного изображения в двумерное распределение гемоглобинового индекса по формуле (2);

- выбор модели спектра транспортного показателя рассеяния ткани $\mu_{s}^{\prime}(\lambda)$, соответствующей исследуемому объекту;

- вычисление монохроматической передаточной функции $D\left(\lambda_{\mathrm{ex}}, \lambda\right)$ в спектральном диапазоне блока регистрации сигнала флуоресценции по формуле (8) с коэффициентами $a_{i}$, приведенными на рис. 2 ;

- определение корректирующего коэффициента для сигнала флуоресценции, регистрируемого в конечном спектральном интервале, по значению $D\left(\lambda_{\mathrm{ex}}, \lambda\right)$ на эффективной длине волны, задаваемой формулой (9), либо

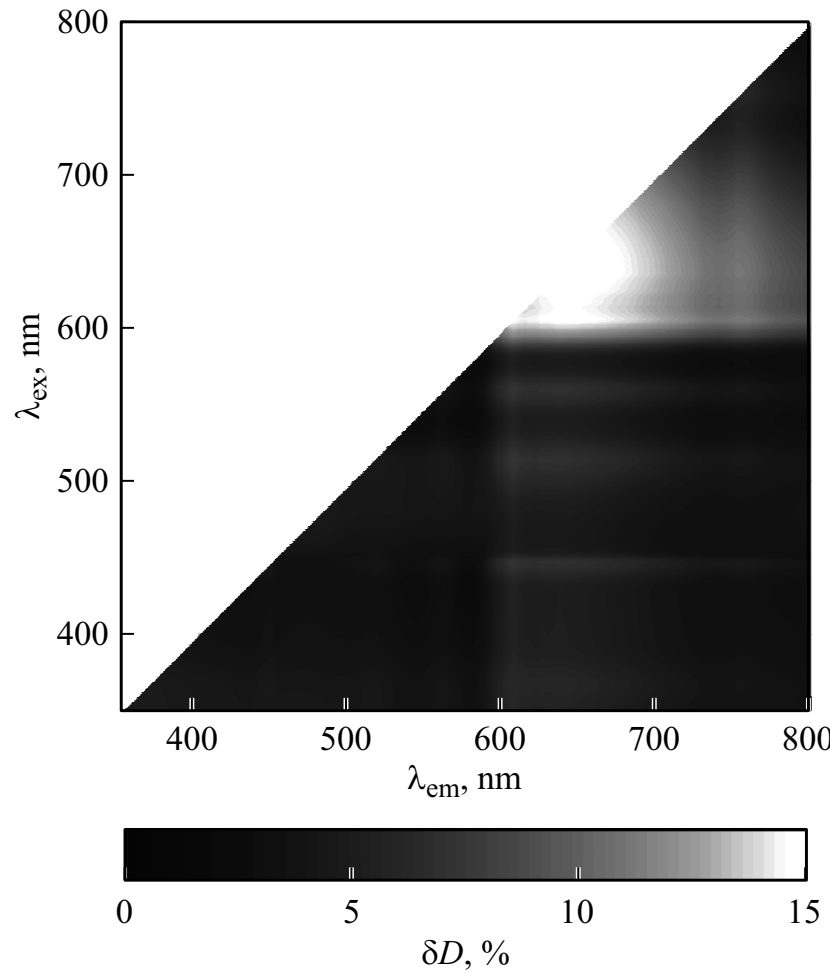

Рис. 3. Относительная среднеквадратическая погрешность вычисления передаточной функции однородной среды, имитирующей биоткань, по формуле регрессии (8).

путем квадратурного суммирования монохроматических значений $D\left(\lambda_{\mathrm{ex}}, \lambda\right)$ с учетом чувствительности фотоприемника $\Phi(\lambda)$.

В алгоритмическом плане разработанная методика не включает сложных математических операций, требующих больших затрат машинного времени, и при ее многопоточной программной реализации на базе графического процессора (GPU) может обеспечивать производительность вычислений, достаточную для работы с изображениями в непрерывном видеорежиме.

\section{Верификация методики}

Эффективность разработанной методики оценим на основе имитационного моделирования флуоресценции биологических объектов методом Монте-Карло. Использовался классический алгоритм моделирования флуоресценции внутри рассеивающей и поглощающей среды [39]. Алгоритм основан на разыгрывании многочисленных случайных траекторий фотонов в среде, включая свободный пробег фотона между рассеивающими центрами среды, учет поглощения фотона и определение нового направления его движения после рассеяния в среде, отражение и преломление на границе раздела сред. Выбор между процессами рассеяния и поглощения фотона, возбуждающего флуоресценцию, осуществляется в соответствии с результатом сравнения альбедо 

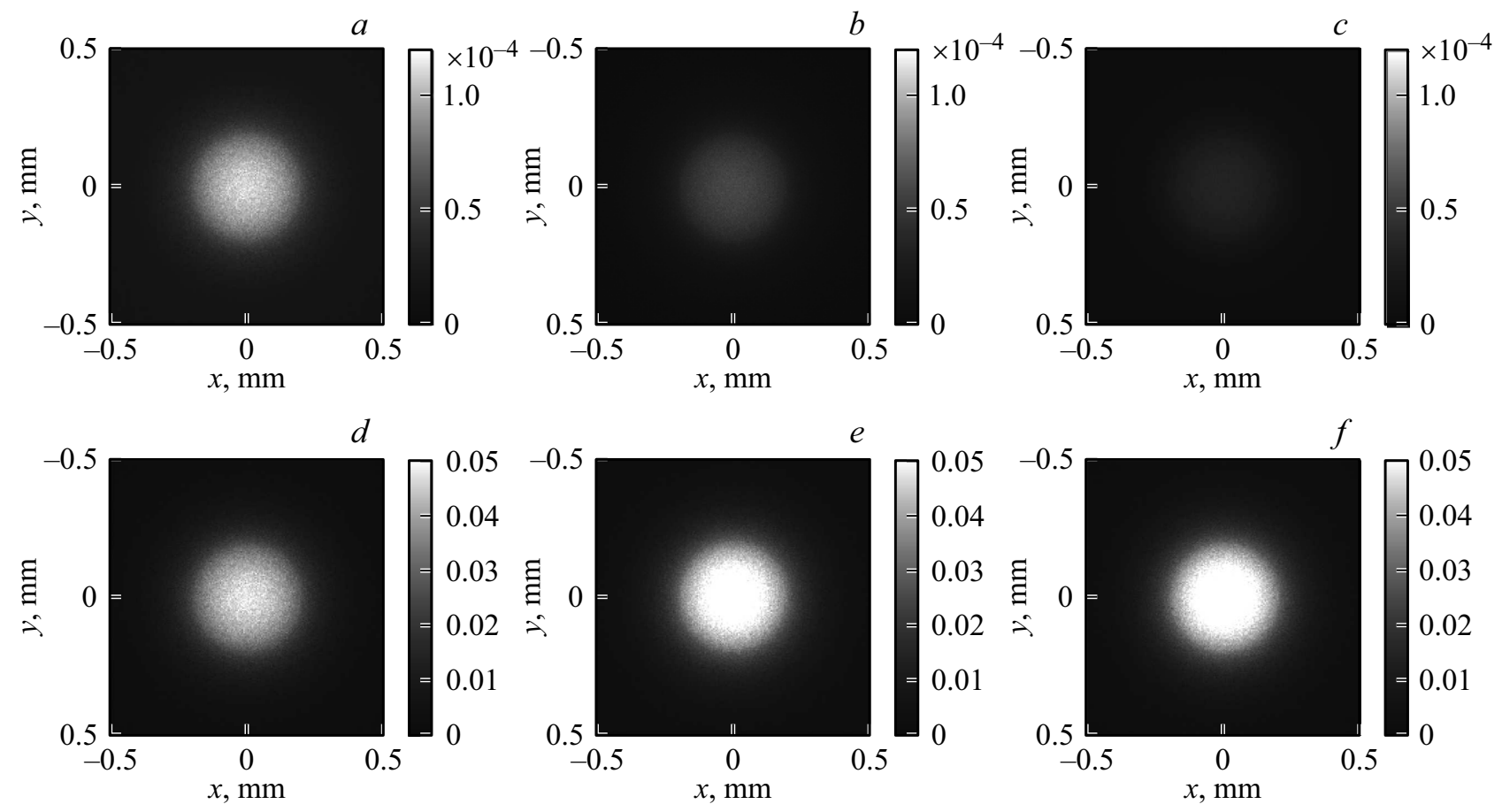

Рис. 4. Результаты коррекции изображений фантомов ткани, смоделированных методом Монте-Карло при объемных концентрациях кровеносных сосудов $0.02(a, d), 0.05(b, e)$ и $0.1(c, f) ; a-c$ - исходные изображения, $d-f-$ скорректированные изображения.

однократного рассеяния среды со случайно сгенерированным числом из диапазона от 0 до 1 . В качестве модели индикатрисы рассеяния света в среде использовалась широко известная функция Хэньи-Гринстейна [40], отражающая главное свойство индикатрис рассеяния оптически мягких частиц с размером порядка длины волны света - сильную вытянутость вперед.

С целью ускорения вычислений предполагалось, что каждый поглощенный фотон внешнего возбуждающего излучения „рождает“ фотон флуоресценции, которому присваивается начальный вес 1.0. Траектории „рожденных“ фотонов отслеживаются аналогично их предшественникам с тем отличием, что вес вторичного фотона последовательно уменьшается после каждого его акта рассеяния пропорционально альбедо однократного рассеяния среды на длине волны из области ее флуоресценции. Распределение фотонов флуоресценции по длинам волн моделируется в соответствии со спектральными зависимостями их квантового выхода $Q_{f}(\lambda)$ и чувствительностью фотоприемного устройства $\Phi(\lambda)$.

При моделировании изображений среды методом Монте-Карло фотоны „запускались“ по нормали к ее поверхности в пределах площадки $1.5 \times 1.5 \mathrm{~mm}$. Общее количество рассмотренных траекторий фотонов $3 \cdot 10^{9}$. Веса всех фотонов флуоресценции, вылетающих из центральной части освещаемой площадки размером $1.0 \times 1.0 \mathrm{~mm}$, регистрировались в ячейках двумерного массива, соответствующих пространственному разрешению на поверхности среды $5 \mu \mathrm{m}$.
Рассмотрено четыре фантома ткани с одинаковыми рассеивающими и с различными поглощающими свойствами. Транспортный показатель рассеяния и средний косинус индикатрисы рассеяния модельных сред аналогичны ткани желудка человека, лабораторно исследованной в работе [41] на основе интегрирующих сфер. Спектры их показателей поглощения описываются формулами (5)-(7) при $D_{V}=40 \mu \mathrm{m}, S=0.85$ и $f_{V}=0.02,0.05$, 0.1 . В качестве флуоресцирующей субстанции выбран 5-АЛК-индуцированный протопорфирин IX (ПП IX), спектр поглощения и флуоресценции которого хорошо известен [42]. Флуорофор был равномерно распределен в цилиндрическом объеме диаметром $400 \mu \mathrm{m}$ и высотой $200 \mu \mathrm{m}$ на глубине $200 \mu \mathrm{m}$ от поверхности среды. Полагалось, что флуорофор возбуждается монохроматическим излучением с длиной волны $405 \mathrm{~nm}$ и излучает в диапазоне 610-700 nm. Спектральная чувствительность блока регистрации фотонов флуоресценции соответствовала красному каналу ПЗС-матрицы ICX285AQ фирмы Sony (Япония). Сигналы отраженного света рассчитывались методом Монте-Карло в спектральном диапазоне $400-700 \mathrm{~nm}$ с разрешением $5 \mathrm{~nm}$ и интегрировались с весовыми функциями, соответствующими спектральным чувствительностям трех цветовых каналов ПЗС-матрицы.

Изображения фантомов биоткани, построенные по результатам Монте-Карло моделирования, показаны на рис. 4, $a-c$. Видно, что увеличение содержания гемоглобина в образцах ткани приводит к существенному падению яркости их флуоресценции: средние яркости 

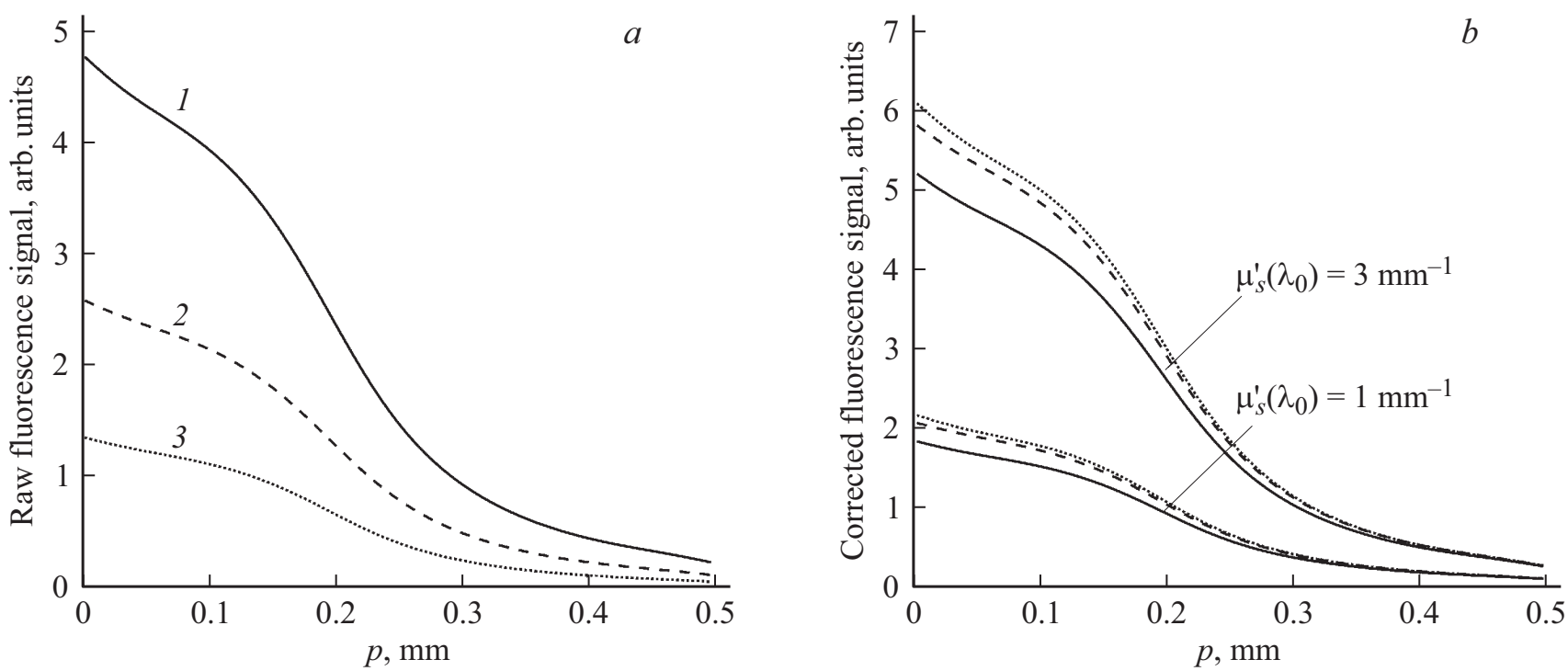

Рис. 5. Радиальные распределения яркостей флуоресцентных изображений, смоделированных методом Монте-Карло (a), и результаты их коррекции при двух априорно заданных значениях транспортного показателя рассеяния среды $(b)$.

центральных пятен диаметром $400 \mu \mathrm{m}$ (область локализации флуорофора) для фантомов ткани с объемными концентрациями кровеносных сосудов $f_{V}=0.02$, 0.05 и 0.1 соотносятся между собой как 1:0.54:0.28. При флуоресцентной диагностике с участием сенсибилизатора это обстоятельство может сильно затруднять обнаружение злокачественных опухолей ввиду более высокой плотности их сосудистой сети по сравнению со здоровой тканью. Очевидно, что без надлежащей коррекции флуоресцентных изображений, регистрируемых в непрерывном видеорежиме, невозможно говорить об установлении каких-либо пороговых значений яркости флуоресценции для нормальных и поврежденных раком тканей и их использовании для создания автоматизированных компьютерных систем поддержки принятия решений в эндоскопии.

Рассмотрим, как изменится видимость очагов флуоресценции после коррекции изображений. Спектр транспортного показателя рассеяния образцов ткани, используемый в качестве априорной информации для нахождения их передаточной функции, описывался формулой (4) при $\mu_{s}^{\prime}\left(\lambda_{0}\right)=2.0$ и $v=1.5$. Их гемоглобиновые индексы определялись по цветовым составляющим изображений в отраженном видимом свете [26]. Корректирующие коэффициенты рассчитаны по формуле (8) для значения $\lambda_{\mathrm{em}}$, усредненного по полосе флуоресценции ПП IX c учетом спектральной чувствительности красного канала ПЗС-матрицы.

Результаты коррекции рассматриваемых изображений показаны на рис. $4, d-f$. Видно, что яркости очагов флуоресценции трех фантомов ткани в значительной мере выровнены: соотносятся между собой как $1: 1.1: 1.15$ при соотношении соответствующих им значений $f_{V}$ $0.02: 0.05: 0.1$. Такой результат коррекции практически равносилен флуоресцентной визуализации ткани при отсутствии в ней поглощения света. В этом случае можно считать, что регистрируемый сигнал флуоресценции пропорционален концентрации сенсибилизатора в биоткани.

Стоит заметить, что выбор модели спектра $\mu_{s}^{\prime}(\lambda)$ при вычислении корректирующих коэффициентов приводит лишь к изменению общего уровня сигналов флуоресценции для всех фантомов ткани, практически не сказываясь на соотношении между величинами этих сигналов. Это особенно видно по радиальным распределениям яркостей исходных и скорректированных флуоресцентных изображений для образов ткани с различным содержанием кровеносных сосудов (рис. 5). Трехкратное увеличение величины $\mu_{s}^{\prime}(\lambda)$, априорно задаваемой при коррекции изображений, приводит к примерно такому же усилению их яркости, но соотношение между яркостями скорректированных изображений различных образцов ткани от этого практически не изменяется. Таким образом, для сравнительного анализа степени накопления сенсибилизатора в тканях по яркости их флуоресценции вполне достаточно учета лишь поглощающей способности ткани, связанной с присутствием в ней гемоглобина, а рассеивающие свойства исследуемого типа биоткани можно учитывать с использованием характерной для нее модели $\mu_{s}^{\prime}(\lambda)$.

\section{Заключение}

Представленные в работе результаты составляют основу новой методики оперативной коррекции флуоресцентных изображений биотканей на эффекты ослабления в них света. Методика может быть легко реализована на базе современной эндоскопической аппаратуры с 
возможностью съемки в отраженном видимом свете и в свете флуоресценции. Ее наиболее важные достоинства: простота программной реализации, малые вычислительны затраты (при использовании графических процессоров, позволяющих осуществлять многопотоковые параллельные вычисления, возможна коррекция изображений в режиме реального времени), получение всех данных, необходимых для расчета передаточной функции среды, непосредственно по регистрируемым изображениям. Как показано на модельных образцах биоткани, скорректированные флуоресцентные изображения достаточно адекватно отражают содержание флуорофора в среде, что открывает возможности для разработки различных пороговых критериев флуоресцентной диагностики новообразований в тканях человека.

\section{Список литературы}

[1] Ramanujam N. Fluorescence Spectroscopy in vivo. / Encyclopedia of Analytical Chemistry. / Ed. by Meyers R. Chichester: John Wiley and Sons, 2000. P. 20-56. doi 10.1002/9780470027318.a0102

[2] Соколов В.В., Русаков И.Г., Булгакова Н.Н., Ульянов Р.В., Теплова А.А. // Сибирский онкологический журн. 2007. № 4 (24). C. 117.

[3] Ширяев А.А., Мусаев Г.Х., Лощенов М.В., Бородкин А.В., Левкин В.В., Охотникова Н.Л., Волков В.В., Макаров В.И., Лощенов В.Б. // Biomedical Photonics. 2016. Т. 5. № 4. C. 15.

[4] Qu J.Y., MacAulay C.E., Lam S., Palcic B. // Opt. Eng. 1995. V. 34. N 11. P. 3334. doi $10.1117 / 12.212917$

[5] Zonios G.I., Cothren R.M., Arendt J.T., Wu J., Van Dam J., Crawford J.M., Manoharan R., Feld M.S. // IEEE Trans. Biomed. Eng. 1996. V. 43. N 2. P. 113. doi 10.1109/10.481980

[6] Zhu B., Sevick-Muraca E.M. // Br. J. Radiol. 2015. Jan. V. 88(1045). P. 20140547. doi 10.1259/bjr.20140547

[7] Valdés P.A., Leblond F., Jacobs V.L., Wilson B.C., Paulsen K.D., Roberts D.W. // Sci. Rep. 2012. V. 2. P. 798. doi 10.1038/srep00798

[8] Xiea Y., Thomb M., Miserocchic A., McEvoyc A.W., Desjardinsd A., Ourselina S., Vercauterena T. // Proc. SPIE. 2017. V. 10050. P. 1005002. doi 10.1117/12.2250159

[9] Themelis G., Yoo J.S., Soh K.S., Schulz R., Ntziachristos V. // J. Biomed. Opt. 2009. V. 14. N 6. P. 064012. doi $10.1117 / 1.3259362$

[10] Lin W.C., Toms S.A., Jansen E.D., Mahadevan-Jansen A. // IEEE J. Sel. Topics Quant. Electron. 2001. V. 7. N 6. P. 996. doi 10.1109/2944.983305

[11] Sinaasappel M., Sterenborg H.J.C.M. // Appl. Opt. 1993. V. 32. N 4. P. 541. doi 10.1364/AO.32.000541

[12] Bogaards A., Sterenborg H.J.C.M., Trachtenberg J., Wilson B.C., Lilge L. // Lasers. Surg. Med. 2007. V. 39. N 7. P. 605. doi $10.1002 / 1 \mathrm{sm} .20525$

[13] Xie H., Liu H., Svenmarker P., Axelsson J., Xu C.T., Gräfe S., Lundeman J.H., Cheng H.P.H., Svanberg S., Bendsoe N., Andersen P.E., Svanberg K., Andersson-Engels S. // J. Biomed. Opt. 2011. V. 16. N 6. P. 066002. doi $10.1117 / 1.3585675$

[14] Sinichkin Yu.P., Utz S.R., Mavliutov A.H., Pilipenko H.A. // J. Biomed. Opt. 1998. V. 3. N 2. P. 201. doi 10.1117/1.429876
[15] Chang S.K., Arifler D., Drezek R., Follen M., RichardsKortum R. // J. Biomed. Opt. 2004. V. 9. N 3. P. 511. doi $10.1117 / 1.1695559$

[16] Kokhanovsky A.A. // J. Opt. Soc. Am. A. 2009. V. 26. N 8. P. 1896. doi 10.1364/JOSAA.26.001896

[17] Kim A., Khurana M., Moriyama Y., Wilson B.C. // J. Biomed. Opt. 2010. V. 15. N 6. P. 067006. doi $10.1117 / 1.3523616$

[18] Рогаткин Д.А., Смирнова О.Д. // Оптический журнал. 2013. T. 80. № 9. C. 54.

[19] Gardner C.M., Jacques S.L., Welch A.J. // Appl. Opt. 1996. V. 35. N 10. P. 1780. doi 10.1364/AO.35.001780

[20] Yudovsky D., Pilon L. // Appl. Opt. 2010. V. 49. N 31. P. 6072. doi 10.1364/AO.49.006072

[21] Saager R.B., Cuccia D.J., Saggese S., Kelly K.M., Durkin A.J. // J. Biomed. Opt. 2011. V. 16. N 12. P. 126013. doi 10.1117/1.3665440

[22] Yang B., Sharma M., Tunnell J.W. // J. Biomed. Opt. 2013. V. 18. N 8. P. 080503. doi 10.1117/1.JBO.18.8.080503

[23] Yang B., Tunnell J.W. // J. Biomed. Opt. 2014. V. 19. N 9. P. 090505. doi 10.1117/1.JBO.19.9.090505

[24] Kress J., Rohrbach D.J., Carter K.A., Luo D., Shao S., Lele S., Lovell J.F., Sunar U. // Biomed. Opt. Express. 2015. V. 6. N 9. P. 3546. doi 10.1364/BOE.6.003546

[25] Лысенко С.А. // Опт. и спектр. 2018. Т. 124. № 1. С. 129; Lysenko S.A. // Opt. Spectrosc. 2018. V. 124. N 1. P. 129-139.

[26] Лысенко С.А. // Журн. прикл. спектр. 2017. Т. 84. № 3. C. 419; Lisenko S.A. // J. Appl. Spectrosc. 2017. V. 84. N 3. P. 439. doi 10.1007/s10812-017-0489-0

[27] Kanick S.C., Davis S.C., Zhao Y., Hasan T., Maytin E.V., Pogue B.W., Chapmand M.S. // J. Biomed. Opt. 2014. V. 19. N 7. P. 075002. doi $10.1117 / 1 . J B O .19 .7 .075002$

[28] Лысенко С.А., Фираго В.А., Кугейко М.М., Кубарко А.И. // Журн. прикл. спектр. 2016. Т. 83. № 4. С. 606; Lisenko S.A., Firago V.A., Kugeiko M.M., Kubarko A.I. // J. Appl. Spectrosc. 2016. V. 83. N 4. P. 617. doi 10.1007/s10812-016-0337-7

[29] Зеге Э.П., Иванов А.П., Кацев И.Л. Перенос изображения в рассеивающей среде. Минск: Наука и техника, 1985. $327 \mathrm{c}$.

[30] Розенберг Г.В. // УФН. 1967. Т. 91. Вып. 4. С. 569.

[31] Van Gemert M.J.C., Jacques S.L., Sterenborg H.J., Star W.M. // EEE Trans. Biomed. Engin. 1989. V. 46. N 12. P. 1146. doi 10.1109/10.42108

[32] Лысенко С.А., Кугейко М.М. // Измерит. техн. 2013. № 6. C. 67; Lisenko S.A., Kugeiko M.M. // Meas. Tech. 2013. V. 56. N 6. P. 721. doi 10.1007/s11018-013-0271-5

[33] Лысенко С.А., Кугейко М.М. // Измерит. техн. 2013. № 11. C. 68; Lysenko S.A., Kugeiko M.M. // Meas. Tech. 2014. V. 56. N 11. P. 1302. doi 0.1007/s11018-014-0372-9

[34] Лысенко С.А., Кугейко М.М., Фираго В.А., Собчук А.Н. // Журн. прикл. спектр. 2014. T. 81. № 1. C. 128; Lisenko S.A., Kugeiko M.M., Firago V.A., Sobchuk A.N. // J. Appl. Spectrosc. 2014. V. 81. N 1. P. 118. doi 10.1007/s10812-014-9896-7

[35] Van Veen R.L.P., Verkruysse W., Sterenborg H.J.C.M. // Opt. Lett. 2002. V. 27. N 4. P. 246. doi 10.1364/OL.27.000246

[36] Bargo P.R., Prahl S.A., Goodell T.T., Sleven R.A., Koval G., Blair G., Jacques S.L. // J. Biomed. Opt. 2005. V. 10. N 3. P. 034018. doi 10.1117/1.1921907

[37] Bashkatov A.N., Genina E.A., Tuchin V.V. // J. Innovative Opt. Health Sci. 2011. V. 4. N 1. P. 9. doi $10.1142 / \mathrm{S} 1793545811001319$ 
[38] Jacques S.L. // Phys. Med. Biol. 2013. V. 58. N 11. P. R37. doi 10.1088/0031-9155/58/11/R37

[39] Welch A.J., Gardner C., Richards-Kortum R., Chan E., Criswell G., Pfefer J., Warren S. // Lasers. Surg. Med. 1997. V. 21. N 2. P. 166.

[40] Henyey L.G., Greenstein J.L. // Astrophys. J. 1941. V. 93. P. 70. doi 10.1086/144246.

[41] Bashkatov A.N., Genina E.A., Kochubey V.I., Gavrilova A.A., Kapralov S.V., Grishaev V.A., Tuchin V.V. // Medical Laser Appl. 2007. V. 22. N 2. P. 95. doi 10.1016/j.mla.2007.07.003

[42] Rollakanti K.R., Kanick S.C., Davis S.C., Pogue B.W., Maytin E.V. // Photon. Lasers Med. 2013. V. 2. N 4. P. 287. doi 10.1515/plm-2013-0030 